\title{
Digit Ratio (2D:4D) and Attachment Styles in Middle Childhood: Indirect Evidence for an Organizational Effect of Sex Hormones
}

\author{
Marco Del Giudice ${ }^{1} \cdot$ Romina Angeleri $^{1}$
}

Received: 13 March 2015 /Revised: 1 May 2015 /Accepted: 4 May 2015 /

Published online: 13 May 2015

C) Springer International Publishing 2015

\begin{abstract}
Sex differences in attachment are absent during infancy and early childhood, emerge in middle childhood with self-reports and doll-play tasks, and persist into adulthood, when they are most reliably detected in romantic attachment styles. In our previous work, we hypothesized that sex differences in attachment develop under the influence of adrenal androgens during the transition form early to middle childhood, following activation of hormone-sensitive neural pathways organized by prenatal and early postnatal exposure to sex hormones. In this study we tested the association between the right-hand 2D:4D digit ratio (a marker of early exposure to androgens and estrogen) and sexually differentiated dimensions of attachment in middle childhood assessed with the Coping Strategies Questionnaire (CSQ). In a sample of 285 Italian children aged 8-10 years, females scored lower in avoidance and higher in preoccupation, while no significant sex differences were observed in felt security. Consistent with our predictions, higher (feminized) digit ratios were significantly associated with lower avoidance and higher preoccupation scores in both males and females. In contrast, there was no significant association between digit ratio and felt security in either sex. These results corroborate the hypothesis that sex differences in attachment reflect the activation of sexually differentiated pathways organized in early development, and for the first time implicate sex hormones in the development of individual differences in attachment styles.
\end{abstract}

Keywords 2D:4D $\cdot$ Androgens $\cdot$ Attachment $\cdot$ Digit ratio $\cdot$ Middle childhood $\cdot$ Sex differences

Electronic supplementary material The online version of this article (doi:10.1007/s40750-015-0027-3) contains supplementary material, which is available to authorized users.

Marco Del Giudice marcodg@unm.edu

1 Department of Psychology, University of New Mexico, Logan Hall, 2001 Redondo Dr. NE, Albuquerque, NM 87131, USA 


\section{Introduction}

Attachment theory is a powerful framework for understanding the formation, development, and dissolution of close relationships, from the initial bond between infant and caregiver to sexual and romantic relations between adult partners. Individual differences in attachment styles have been linked to an impressive range of correlates spanning neurobiology, cognitive and affective processes, social and sexual behavior, and psychopathology (see Cassidy and Shaver 2008; Mikulincer and Shaver 2007). Until very recently, attachment researchers have paid little attention to sex differences, based on the classic finding that patterns of attachment in infancy and early childhood have essentially the same distribution in males and females (reviewed in Del Giudice 2009). However, romantic attachment styles in adulthood have been shown to differ systematically between the sexes (Del Giudice 2011; Schmitt et al. 2003). Across countries, women tend to display lower levels of avoidance (e.g., self-reliance, discomfort with emotional closeness) and higher levels of anxiety (e.g., emotional neediness, preoccupation with rejection and abandonment). When attachment "states of mind" are coded as discrete categories from interviews, no sex differences can be detected (Bakermans-Kranenburg and van IJzendoorn 2010); however, when interviews are used to generate dimensional scores, a pattern consistent with that observed in romantic attachment emerges, with women scoring significantly lower than men in dismissiveness (a dimension closely related to avoidance) and higher than men in preoccupation (a dimension closely related to anxiety; Haydon et al. 2014).

When do these sex differences emerge in development? Del Giudice (2009) reviewed the available literature and concluded that attachment styles appear to become sexually differentiated during the transition from early to middle childhood. Subsequent studies of attachment in middle childhood employing self-report questionnaires and doll-play tasks have consistently found lower levels of avoidance and higher levels of preoccupation in girls (e.g., Chen and Chang 2012; Kerns et al. 2011; Toth et al. 2013). In contrast, attachment interviews tailored to middle childhood have failed to reveal significant sex differences (e.g., Bakersman-Kranenburg and van IJzendoorn 2009; Shmueli-Goetz et al. 2008; Venta et al. 2014).

The discrepancy between interviews on one side and questionnaires and doll-play tasks on the other might be due to a number of factors. To begin with, both questionnaires and doll-play tasks focus on overt behavior (e.g., self-reliance, distancing of parents, separation anxiety); in contrast, interviews center on the child's representations of attachment figures, and their coding systems emphasize narrative qualities such as coherence, emotional openness, and idealization rather than descriptions of attachment-related behavior (Shmueli-Goetz et al. 2008; Venta et al. 2014). To the extent that sex differences concern the behavioral regulation of attachment relationships, they may be difficult to capture with current interview protocols. Of note, self-reports and doll-play tasks are systematically associated with measures of peer functioning (e.g., Finnegan et al. 1996; Granot and Mayseless 2001; Waniel et al. 2008), whereas childhood interviews show only weak associations with peer relationships (Venta et al. 2014). This finding suggests that self-reports may be tapping into aspects of behavior that are missed by interviews. In addition, the coding systems of childhood interviews mirror the discrete categories of adult interviews. Recent evidence from studies of adults (Haydon et al. 2014) suggests 
that the categorical coding of attachment states of mind may obscure the existence of sex differences. It is possible that dimensional scores similar to those generated by Haydon et al. (2014) would reveal reliable sex differences in childhood interviews as well.

Del Giudice and colleagues argued that sex differences in attachment behavior emerging in middle childhood can be explained by social and sexual selection in the context of heightened competition for status and social resources, as well as by sex differences in optimal reproductive strategies (Del Giudice 2009, in press; Del Giudice and Belsky 2010). In a nutshell, avoidant styles are correlated with self-reliance, "pseudo-maturity", and aggression; in boys, this constellation of traits can be especially adaptive in view of risky competition for status and dominance. In adulthood, attachment avoidance minimizes commitment in close relationships and supports investment in short-term sexual relationships. In females, successful reproduction primarily requires securing sufficient material and social resources (for example a supportive network of relatives and friends). Anxious attachment emphasizes neediness and vulnerability, and is characterized by constant preoccupation about the availability and commitment of one's partners. For these reasons, anxiety contributes to maximize investment of time and resources by relatives and partners - especially if the latter tend to be unreliable or uncommitted.

On this view, males and females should primarily differ in their typical styles of insecure attachment - associated with stressful environments and harsh family relationships - but not in their overall levels of security. Also, sex differences in attachment styles are predicted to be relatively small in safe, supportive ecological conditions, increase at moderate levels of stress, and decrease again at very high levels of harshness and unpredictability. In highly dangerous environments, both sexes should benefit from low-investment reproductive strategies consistent with avoidant styles (discussed in Del Giudice 2009; Del Giudice and Belsky 2010). Finally, Del Giudice (2011) speculated that avoidant attachment may sometimes have different implications in men and women; for example, preoccupied women might show temporary elevations in avoidance following periods of acute relational stress (for details see Del Giudice 2009, 2011).

Del Giudice and colleagues further proposed that the emergence of sex differences in middle childhood is part of a broader developmental transition mediated by adrenarche. Adrenarche (or "adrenal puberty") is the onset of production of androgens such as dehydroepiandrosterone (DHEA) and dehydroepiandrosterone sulfate (DHEAS) by the adrenal gland, and occurs in both sexes between 6 and 8 years of age (Del Giudice 2009, 2014; Del Giudice et al. 2009). Notably, adrenal androgens can be converted to testosterone and/or estrogen in the brain (Labrie et al. 1998, 2001). Del Giudice and colleagues speculated that adrenarche plays a key role in the activation of sexually differentiated neurobiological pathways. These pathways would be organized by sex hormones during fetal development and early infancy, and remain dormant through early childhood until they are activated by rising concentrations of adrenal androgens (and/or testosterone and estrogen) in middle childhood. The interaction between the early organizational effects of sex hormones and the activational effects of adrenal androgens would contribute to explain not only the average pattern of differences between the sexes, but also individual variation in attachment styles within each sex. For example, insecurely attached children of both sexes that have been exposed to a high androgen/estrogen ratio should be comparatively more likely to develop male-typical profiles of attachment (high avoidance, low anxiety) when they enter middle childhood. 
In summary, there is evidence that sex differences in attachment become apparent starting from middle childhood (at least in self-report questionnaires and doll-play tasks), and theoretical considerations point to a likely involvement of sex hormones at multiple time points. To our knowledge, however, no study has yet investigated the association between attachment styles and indices of sex hormones activity. In the present study we aimed to provide an initial test of this hypothesized association by correlating attachment styles in middle childhood with individual differences in the 2D:4D digit ratio, an anatomical marker of early exposure to sex hormones.

\section{The 2D:4D Digit Ratio as a Marker of Early Exposure to Sex Hormones}

The relative length of the second to fourth digit (2D:4D digit ratio) is a sexually dimorphic trait in humans and many other mammalian species. The fourth digit tends to be shorter in females; as a result, females have higher digit ratios than males. Digit ratios develop prenatally, and are partly determined by the balance of androgen and estrogen signaling during the time window for digit development in the fetus (see Berenbaum et al. 2009; Manning et al. 2014; Zheng and Cohn 2011). While the prenatal origin of digit ratios has been established conclusively, there is suggestive evidence that - at least in humans — sex hormones levels in early infancy may further contribute to individual and sex differences in relative finger length (Galis et al. 2010; Knickmeyer et al. 2011). In childhood, sex differences in 2D:4D can be reliably detected from at least 5 years of age, though they are somewhat smaller than those observed in adults (McIntyre et al. 2005; McIntyre et al. 2006; Trivers et al. 2006).

The small but reliable association between relative finger length and prenatal androgen/estrogen balance makes the digit ratio a viable biomarker for early exposure to sex hormones. The gold standard for digit ratio measurement is based on bone length estimation from X-rays, CT scans, or similar methods (e.g., McIntyre et al. 2005); however, most studies in humans measure digit ratios indirectly on the skin surface, either with a caliper or from hand scans or photocopies. While indirect methods are considerably more practical, they increase the measurement error associated with 2D:4D estimation and further weaken the correlation with early hormonal exposure. For all these reasons, associations between digit ratios and behavioral traits can be expected to be rather small in size; a notable exception is the association between 2D:4D and measures of athletic prowess, with a meta-analytic effect size of about $r=$ -0.26 (Hönekopp and Schuster 2010).

\section{Study Hypotheses}

In this study we set out to test two hypotheses. First we predicted that, in a middle childhood sample, girls would show lower levels of avoidance and higher levels of preoccupation than boys (consistent with previous research). Second, we predicted that digit ratios would be associated with individual differences in attachment within sex; in particular, we expected higher (feminized) digit ratios to correlate with lower avoidance and higher preoccupation-that is, more female-typical attachment styles. 


\section{Method}

\section{Participants}

Participants were 285 Italian children (135 males, 150 females) aged 8.1 to 10.9 years ( $M=113.0$ months, $S D=7.2$ months). Children were recruited from schools and summer camps in Northern Italy and tested on-site after obtaining parental consent. The study was approved by the IRB of the University of Turin (Torino, Italy), to which the authors were affiliated at the time of data collection. The age window of 8-10 years was chosen to maximize the likelihood that participants would have undergone adrenarche (the onset of androgen production by the adrenal glands) but not gonadarche (the onset of estrogen/androgen production by the ovaries/testes). The available data show that the median age at menarche in Northern Italy is 12.4 years, with $95 \%$ of girls reaching menarche at 10.4 years or later (Rigon et al. 2010.)

\section{Materials and Procedure}

Digit Ratio Each participant's right hand was digitally scanned with a resolution of 300 dpi with a Canon ${ }^{\mathrm{TM}}$ LiDE $^{\mathrm{TM}} 35$ flatbed scanner, and saved in an uncompressed TIFF color image file. Participants were instructed to place their hands on the scanner, slightly spreading their fingers. Images were inspected immediately after scanning, and images of poor quality (because of movement artifacts, incorrect hand positioning, and so on) were re-acquired until satisfactory quality was obtained. The right hand was chosen because it shows larger sex differences in digit ratio, and is therefore likely to provide a better indicator of early exposure to sex hormones (Hönekopp and Watson 2010).

The second and fourth finger were measured with the image processing software ImageJ (Rasband 1997-2014) on a 21-in. display by one of the authors, who was blind to the sex and attachment scores of participants. Following standard practice, finger length was measured from the fingertip to the midpoint of the ventral proximal crease (Voracek et al. 2007). The length of the second finger was divided by that of the fourth finger and rounded to three decimal points to yield the 2D:4D digit ratio. Computerassisted measurement of digit ratios has been shown to be consistently more reliable than physical measurement and measurement from photocopies or printed scans (Allaway et al. 2009; Kemper and Schwerdtfeger 2009). As a reliability check, 100 randomly sampled images were independently measured by a trained research assistant; the intraclass correlation for digit ratios was $0.91(p<0.001)$, indicating highly reliable measurement.

Attachment Styles Attachment styles were assessed with the revised version of the Coping Styles Questionnaire (CSQ; Yunger et al. 2005; see Finnegan et al. 1996). The questionnaire consists of 28 short vignettes, for example: "You are at the movies with your mother and you have to go out to the bathroom. When you come back in, the theatre is so dark that you can't find your mother." After each vignette, two alternatives are presented, for example: "Some kids would calmly look for their mother and not be too worried; BUT other kids would look for their mother and be very upset until they found her." Children are asked to choose the option that would best represent their behavior as either "sort of true for me" or "very true for me." The CSQ has three 
subscales: a 10-item avoidance subscale with scores ranging from 0 to 20 , a 10 -item preoccupation subscale with scores ranging from 0 to 20 , and an 8 -item felt security subscale with scores ranging from 0 to 16 .

The questionnaire was independently translated into Italian by each of the present authors; the two translations were compared and discrepancies were discussed, yielding a final Italian version of the questionnaire (see the Supplementary Material). Parallel analysis of item correlations suggested the presence of three reliable components, which were extracted with principal component analysis (PCA) and Oblimin-rotated. The resulting component structure accurately reproduced the three subscales of the original CSQ. Specifically, the first component showed positive loadings of preoccupation items (average of pattern loadings $=0.57$ ), but not avoidance (average $=-0.06$ ) or security items (average $=0.02$ ). The second component showed positive loadings of avoidance items (average $=0.52$ ), but not preoccupation (average $=-0.04$ ) or security items (average $=-0.01$ ). Finally, the third component showed positive loadings of security items (average $=0.53$ ), but not preoccupation (average $=0.03$ ) or avoidance items (average $=-0.01$ ). Internal consistency was $\alpha=0.74$ for the avoidance subscale, $\alpha=0.80$ for preoccupation, and $\alpha=0.68$ for felt security. The correlation between the avoidance and preoccupation subscales was -0.45 , in line with the original CSQ ( $r=$ -0.47 in Finnegan et al. 1996).

\section{Results}

Data analysis was performed in SPSS ${ }^{\mathrm{TM}} 22$ (IBM $^{\mathrm{TM}}$ Corporation) and R 2.15 (Core Team 2012). Descriptive statistics and correlations between study variables in the male and female subsamples are shown in Table 1. As expected, the mean digit ratio was significantly higher in females $\left(t_{(283)}=2.177, p=0.030\right)$. Consistent with previous studies, girls scored significantly lower in avoidance $\left(t_{(283)}=-4.458, p<0.001\right)$ and higher in preoccupation $\left(t_{(283)}=5.099, p<0.001\right)$ than boys. Sex differences in felt security were small and not significantly different from zero $\left(t_{(283)}=0.309, p=0.757\right)$, in line with the idea that males and females differ in their typical insecurity profiles, but not in overall attachment security (Del Giudice 2009).

Table 1 Zero-order correlations, descriptive statistics, and effect size (ES) of sex differences for the study variables. Correlations in males are above the diagonal; those in females are below the diagonal. Positive values of Cohen's $d$ indicate higher scores in males

\begin{tabular}{|c|c|c|c|c|c|c|c|c|c|}
\hline & \multicolumn{4}{|c|}{ Correlations } & \multicolumn{2}{|l|}{ Males } & \multicolumn{2}{|c|}{ Females } & \multirow{2}{*}{$\begin{array}{l}\text { ES } \\
d\end{array}$} \\
\hline & 1. & 2. & 3. & 4. & $M$ & $S D$ & $M$ & $S D$ & \\
\hline 1. Digit ratio & & -0.13 & 0.18 & -0.07 & 0.953 & 0.029 & 0.960 & 0.028 & -0.25 \\
\hline 2. Avoidance & -0.11 & & -0.45 & -0.07 & 3.39 & 3.70 & 1.77 & 2.31 & 0.53 \\
\hline 3. Preoccupation & 0.10 & -0.45 & & 0.01 & 7.01 & 4.57 & 9.96 & 5.12 & -0.61 \\
\hline 4. Felt security & -0.03 & -0.33 & -0.30 & & 10.41 & 3.05 & 10.46 & 3.00 & -0.04 \\
\hline
\end{tabular}


Table 2 Multiple regression of attachment scores on sex and digit ratio

\begin{tabular}{lrrlrlllll}
\hline Avoidance & $\beta$ & $p$ & Preoccupation & $\beta$ & $p$ & Felt security & $\beta$ & $p$ \\
\hline Step 1 & & & & & & & & \\
$\quad$ Sex (male) & 0.24 & $<0.001$ & Sex (male) & -0.27 & $<0.001$ & Sex (male) & -0.03 & 0.675 \\
$\quad$ Digit ratio & -0.12 & 0.045 & Digit ratio & 0.13 & 0.024 & Digit ratio & -0.05 & 0.376 \\
Step 2 & & & & & & & & \\
$\quad$ Sex & 0.24 & $<0.001$ & Sex & -0.27 & $<0.001$ & Sex & -0.03 & 0.673 \\
$\quad$ Digit ratio & -0.11 & 0.048 & Digit ratio & 0.13 & 0.026 & Digit ratio & -0.05 & 0.386 \\
Sex $\times$ digit ratio & -0.03 & 0.555 & Sex $\times$ digit ratio & 0.03 & 0.627 & Sex $\times$ digit ratio & -0.02 & 0.748 \\
\hline
\end{tabular}

The association between digit ratio and individual differences in attachment was tested by fitting a set of multiple regression models to the data (Table 2). For each of the CSQ subscales, sex and digit ratio were entered as centered predictors in the first step. The partial regression coefficient for the digit ratio represents the net association between digit ratio and attachment, controlling for average sex differences in both digit ratio and attachment scores. In the second step, the interaction between sex and digit ratio was added to the model to evaluate whether the association between attachment and digit ratio was significantly different in the two sexes.

As shown in Table 2, the association between digit ratio and individual differences in attachment closely mirrored the pattern of sex differences reported above. Specifically, higher (feminized) digit ratios predicted lower avoidance and higher preoccupation, but there was no significant association between digit ratio and felt security. Analysis of interaction terms (step 2) showed no evidence of differential effects in males and females (see Fig. 1).

In the multiple regression for avoidance, inspection of residuals showed marked deviations from normality due to the right-skewed distribution of avoidance scores. Fitting the regression model to log-transformed avoidance scores yielded virtually identical results (partial regression coefficient of digit ratio: $\beta=-0.12, p=0.048$ ), suggesting that this finding is robust in the face of assumption violations.
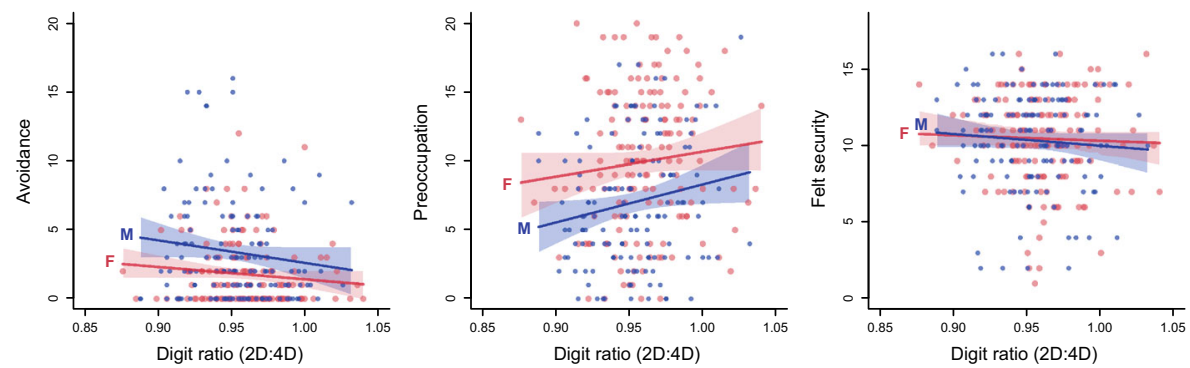

Fig. 1 Scatterplots and linear regression of attachment scores on digit ratio in males (smaller blue dots) and females (larger red dots). Confidence bands show $95 \%$ CIs on regression parameters, computed separately by $\operatorname{sex}$ 


\section{Discussion}

In this study, we hypothesized that (a) in middle childhood, girls would show lower avoidance and higher preoccupation than boys; and that (b) higher (feminized) digit ratios would be associated with lower avoidance and higher preoccupation scores at the individual level. Both hypotheses were supported in our sample. These findings provide initial evidence that early exposure to sex hormones plays an organizational role on the neurobiological pathways implicated in the development of attachment styles. Importantly, the age of children in our sample was selected to fall between the typical age of adrenarche (6-8 years) and the typical age of gonadarche (10-14 years in Northern Italy). Thus, our findings are broadly consistent with the hypothesis that adrenal androgens play an activational role during the transition to middle childhood (Del Giudice 2014; Del Giudice et al. 2009). Of course, the present data do not provide direct evidence of an activational role of adrenal androgens, and are compatible with a number of alternative explanations; for example, the emergence of sex differences might be driven by social changes that co-occur with the transition to middle childhood.

The main limitation of the study lies in the indirect and noisy nature of the digit ratio as a marker of early hormonal exposure. In our study, we strived to maximize the reliability of 2D:4D by employing computer-assisted measurement, which has been shown to be superior to other indirect methods (Allaway et al. 2009; Kemper and Schwerdtfeger 2009). However, it is important to note that a number of other associations between digit ratios and behavioral traits have failed to replicate, especially when the original studies had been conducted in small samples (reviewed in Manning et al. 2014). The reasonable size of our sample and the fact that we found the same pattern of effects in both sexes reduce the likelihood that the present findings are spurious; still, our results should be treated as tentative until replicated or corroborated by other methods. Also, direct measurement of adrenal androgen levels would permit more direct testing of the activational hypothesis and provide further insight on the origin of individual differences in attachment.

In conclusion, we hope that these initial results will stimulate research into the role of sex hormones in the development of attachment styles, and pave the way for stronger integration between evolutionary models of attachment and the study of the underlying neurobiological mechanisms.

\section{References}

Allaway, H. C., Bloski, T. G., Pierson, R. A., \& Lujan, M. E. (2009). Digit ratios (2D:4D) determined by computer-assisted analysis are more reliable than those using physical measurements, photocopies, and printed scans. American Journal of Human Biology, 21, 365-370.

Bakermans-Kranenburg, M. J., \& van IJzendoorn, M. H. (2010). Invariance of adult attachment across gender, age, culture, and socioeconomic status? Journal of Social and Personal Relationships, 27, 200-208.

Bakersman-Kranenburg, M. J., \& van IJzendoorn, M. H. (2009). No reliable gender differences in attachment across the lifespan. Behavioral and Brain Sciences, 33, 22-23.

Berenbaum, S. A., Kormal Bryk, K., Nowak, N., Quigley, C. A., \& Moffat, S. (2009). Fingers as a marker of prenatal androgen exposure. Endocrinology, 150, 5119-5124.

Cassidy, J., \& Shaver, P. R. (Eds.). (2008). Handbook of attachment (2nd ed.). New York: Guilford. 
Chen, B.-B., \& Chang, L. (2012). Adaptive insecure attachment and resource control strategies during middle childhood. International Journal of Behavioral Development, 36, 389-397.

R Core Team (2012). R: A language and environment for statistical computing. R Foundation for Statistical Computing, Vienna, Austria. ISBN 3-900051-07-0, http://www.R-project.org.

Del Giudice, M. (2009). Sex, attachment, and the development of reproductive strategies. Behavioral and Brain Sciences, 32, 1-21.

Del Giudice, M. (2011). Sex differences in romantic attachment: a meta-analysis. Personality and Social Psychology Bulletin, 37, 193-214.

Del Giudice, M. (2014). Middle childhood: an evolutionary-developmental synthesis. Child Development Perspectives, 8, 193-200.

Del Giudice, M. (in press). Attachment in middle childhood: An evolutionary-developmental perspective. New Directions for Child and Adolescent Development.

Del Giudice, M., \& Belsky, J. (2010). Sex differences in attachment emerge in middle childhood: an evolutionary hypothesis. Child Development Perspectives, 4, 97-105.

Del Giudice, M., Angeleri, R., \& Manera, V. (2009). The juvenile transition: a developmental switch point in human life history. Developmental Review, 29, 1-31.

Finnegan, R. A., Hodges, E. V. E., \& Perry, D. G. (1996). Preoccupied and avoidant coping during middle childhood. Child Development, 67, 1318-1328.

Galis, F., Ten Broek, C. M. A., Van Dongen, S., \& Wijnaendts, L. C. D. (2010). Sexual dimorphism in the prenatal digit ratio (2D:4D). Archives of Sexual Behavior, 39, 57-62.

Granot, D., \& Mayseless, O. (2001). Attachment security and adjustment to school in middle childhood. International Journal of Behavioral Development, 25, 530-541.

Haydon, K. C., Roisman, G. I., Owen, M. T., Booth-LaForce, C., \& Cox, M. J. (2014). Shared and distinctive antecedents of adult attachment interview state-of-mind and inferred-experience dimensions. Monographs of the Society for Research in Child Development, 79(3), 108-125.

Hönekopp, J., \& Schuster, M. (2010). A meta-analysis on 2D:4D and athletic prowness: substantial relationships but neither hand out-predicts the other. Personality and Individual Differences, 48, 4-10.

Hönekopp, J., \& Watson, S. (2010). Meta-analysis of digit ratio 2D:4D shows greater sex difference in the right hand. American Journal of Human Biology, 22, 619-630.

Kemper, C. J., \& Schwerdtfeger, A. (2009). Comparing indirect methods of digit ratio (2D:4D) measurement. American Journal of Human Biology, 21, 188-191.

Kerns, K. A., Brumariu, L. E., \& Seibert, A. (2011). Multi-method assessment of mother-child attachment: links to parenting and child depressive symptoms in middle childhood. Attachment \& Human Development, 13, 315-333.

Knickmeyer, R. C., Woolson, S., Hamer, R. M., Kooneker, T., \& Gilmore, J. H. (2011). 2D:4D ratios in the first 2 years of life: stability and relation to testosterone exposure and sensitivity. Hormones and Behavior, 60, 256-263.

Labrie, F., Bélanger, A., Luu-The, V., Labrie, C., Simard, J., Cusan, L., et al. (1998). DHEA and the intracrine formation of androgens and estrogens in peripheral target tissues: its role during aging. Steroids, 6, 322328.

Labrie, F., Luu-The, V., Labrie, C., \& Simard, J. (2001). DHEA and its transformation into androgens and estrogens in peripheral target tissues: intracrinology. Frontiers in Neuroendocrinology, 22, 185-212.

Manning, J., Kilduff, L., Cook, C., Crewther, B., \& Fink, B. (2014). Digit ratio (2D:4D): a biomarker for prenatal sex steroids and adult sex steroids in challenge situations. Frontiers in Endocrinology, 5, e9.

McIntyre, M. H., Ellison, P. T., Lieberman, D. E., Demerath, E., \& Towne, B. (2005). The development of sex differences in digital formula from infancy in the Fels longitudinal study. Proceedings of the Royal Society $B, 272,1473-1479$.

McIntyre, M. H., Cohn, B. A., \& Ellison, P. T. (2006). Sex dimorphism in digital formulae of children. American Journal of Physical Anthropology, 129, 143-150.

Mikulincer, M., \& Shaver, P. R. (2007). Structure, dynamics, and change. New York: Guilford. Attachment in adulthood.

Rasband, W. S. (1997-2014). ImageJ, U. S. National Institutes of Health, Bethesda, Maryland, USA, http:// imagej.nih.gov/ij/.

Rigon, F., Bianchin, L., Bernasconi, S., Ona, G., Bozzola, M., Buzi, F., et al. (2010). Update on age at menarche in Italy: toward the leveling off of the secular trend. Journal of Adolescent Health, 46, 238-244.

Schmitt, D. P., Alcalay, L., Allensworth, M., Allik, J., Ault, L., Austers, I., et al. (2003). Are men universally more dismissing than women? Gender differences in romantic attachment across 62 cultural regions. Personal Relationships, 10, 307-331. 
Shmueli-Goetz, Y., Target, M., Fonagy, P., \& Datta, A. (2008). The child attachment interview: a psychometric study of reliability and discriminant validity. Developmental Psychology, 44, 939-956.

Toth, I., Lakatos, K., \& Gervai, J. (2013). Gender differences in children's responses to attachment story stems: true or artefacts? Bull Int Soc Study Behav Dev, 63, 2-5.

Trivers, R., Manning, J., \& Jacobson, A. (2006). A longitudinal study of digit ratio (2D: 4D) and other finger ratios in Jamaican children. Hormones and Behavior, 49, 150-156.

Venta, A., Shmueli-Goetz, Y., \& Sharp, C. (2014). Assessing attachment in adolescence: a psychometric study of the child attachment interview. Psychological Assessment, 26, 238-255.

Voracek, M., Manning, J. T., \& Dressler, S. G. (2007). Repeatability and interobserver error of digit ratio (2D: 4D) measurements made by experts. American Journal of Human Biology, 19, 142-146.

Waniel, A., Besser, A., \& Priel, B. (2008). Self- and maternal representations, relatedness patterns, and problem behavior in middle childhood. Personal Relationships, 15, 171-189.

Yunger, J. L., Corby, B. C., \& Perry, D. G. (2005). Dimensions of attachment in middle childhood. In K. A. Kerns \& R. A. Richardson (Eds.), Attachment in middle childhood (pp. 89-114). New York: Guilford Press.

Zheng, Z., \& Cohn, M. J. (2011). Developmental basis of sexually dimorphic digit ratios. Proceedings of the National Academy of Sciences of the United States of America, 108, 16289-16294. 